\title{
TRIAGEM AUDITIVA NEONATAL: UM ESTUDO NA CIDADE DE CURITIBA - PR
}

\section{Newborn hearing screening: a study in Curitiba-PR}

\author{
Candice Cristina Stumpf ${ }^{(1)}$, Caroline Gambini ${ }^{(2)}$, Lílian Cássia Bórnia Jacob-Corteletti ${ }^{(3)}$, \\ Simone Mariotto Roggia ${ }^{(4)}$
}

\begin{abstract}
RESUMO
Objetivo: verificar a prática de Triagem Auditiva Neonatal na cidade de Curitiba-PR. Métodos: inicialmente foi realizado um levantamento do número de maternidades e/ou hospitais com maternidades existentes na cidade de Curitiba, mediante a consulta no Cadastro Nacional de Estabelecimentos de Saúde. Constatou-se a existência de um total de 59 hospitais cadastrados, sendo que três destes são maternidades e 18 são hospitais com maternidade. A partir do levantamento feito, foi estabelecido um contato com o profissional responsável por cada uma das instituições, sendo questionado quanto à existência de um programa de Triagem Auditiva Neonatal e para aqueles que o apresentavam foi entregue um questionário a fim de obter informações a respeito do referido serviço. Resultados: constatou-se que apenas $23,8 \%$ das maternidades realizam Triagem Auditiva Neonatal, sendo que destas, $20 \%$ é universal para neonatos a termo e $80 \%$ universal para neonatos de alto risco. A triagem em todos os serviços é realizada com Emissões Otoacústicas Evocadas por Estímulo Transiente. Conclusão: mediante o estudo realizado verificou-se que a lei número 14588 - 22/12/2004 não está sendo rigorosamente cumprida. Apesar da demanda aliada à importância da detecção precoce, a Triagem Auditiva Neonatal não é realizada em todas as maternidades existentes e mesmo naquelas nas quais há um fonoaudiólogo e a triagem auditiva é realizada, esta não é universal.
\end{abstract}

DESCRITORES: Audição; Perda Auditiva; Triagem Neonatal

\section{INTRODUÇÃO}

É consensual o fato de que limitações auditivas interferem de forma significativa nos processos de apropriação da linguagem oral e escrita, uma vez que restrições quanto ao acesso à oralidade exigem adaptações nas diversas relações sociais estabele-

(1) Fonoaudióloga; Clínica de Fonoaudiologia da Universidade Tuiuti do Paraná, UTP, Tuiuti, PR; Mestranda em Distúrbios da Comunicação pela Universidade Tuiuti do Paraná.

(2) Fonoaudióloga; Clínica de Fonoaudiologia da Universidade Tuiuti do Paraná, UTP, Tuiuti, PR; Mestranda em Distúrbios da Comunicação pela Universidade Tuiuti do Paraná.

(3) Fonoaudióloga; Docente do Curso de Fonoaudiologia e do Programa de Pós-Graduação em Distúrbios da Comunicação da Universidade Tuiuti do Paraná, UTP, Tuiuti, PR; Doutora em Distúrbios da Comunicação.

(4) Fonoaudióloga; Docente do Curso de Fonoaudiologia e do Curso de Especialização em Audiologia da Universidade do Vale do Itajaí, UNIVALI, Vale do Itajaí, SC; Doutora em Ciências - Fisiopatologia Experimental.

Conflito de interesses: inexistente cidas pelos sujeitos nem sempre possíveis ${ }^{1}$. Dessa forma, justificam-se iniciativas que contribuam para o avanço teórico-prático voltado ao diagnóstico e intervenção de perdas auditivas o mais precoce possível, sendo estes, decisivos no prognóstico das crianças identificadas com alterações auditivas.

A partir dessa perspectiva, considera-se que toda criança deve ter sua audição avaliada antes dos três meses de idade, com a efetivação do diagnóstico médico e audiológico e início da intervenção fonoaudiológica até os 6 meses de idade ${ }^{2,3}$. É comprovado que a criança que tem o diagnóstico de surdez e início da intervenção fonoaudiológica nesse período terá desenvolvimento de fala e linguagem significantemente melhor do que aquelas identificadas mais tarde ${ }^{4-7}$.

Assim, este período é considerado como o que apresenta maior plasticidade neuronal da via auditiva ${ }^{8} \mathrm{e}$, para que ocorra a maturação das vias auditivas em nível de tronco encefálico, é necessária a estimulação sonora ${ }^{9}$, pois quanto maior a riqueza 
de estímulos, maior as conexões entre a orelha interna e o córtex auditivo ${ }^{8}$.

Nesse contexto, inúmeros programas de Triagem Auditiva Neonatal (TAN) vêm sendo implementados em diversas cidades no país ${ }^{10-12}$, assim como, uma série de protocolos de avaliação têm sido propostos por pesquisadores e comitês da área $2,10,13$.

Considerando-se a invisibilidade da deficiência auditiva, a única maneira de conseguir que a mesma seja diagnosticada até os três meses de idade consiste na realização da Triagem Auditiva Neonatal Universal (TANU), ou seja, todos os bebês devem ser triados, mesmo aqueles sem critérios de risco para a ocorrência de deficiência auditiva ${ }^{14}$.

A TANU constitui-se num excelente veículo para se atingir o objetivo da identificação de todos os bebês com perda auditiva, bem como do início da intervenção antes dos seis meses de idade ${ }^{4}$. A importância da realização da TAN em todos os bebês nascidos nas maternidades deve-se ao fato de que $50 \%$ dos bebês com deficiência auditiva não apresentam fatores de risco para deficiência auditiva ${ }^{14}$. Deste modo, se a TAN for realizada apenas nos bebês com fatores de risco para deficiência auditiva, $50 \%$ dos bebês restantes poderão ter um diagnóstico e intervenção em idades tardias e não aceitáveis ${ }^{14}$.

O avanço tecnológico e os diversos estudos focando o assunto têm possibilitado a utilização de métodos de avaliação objetivos nos programas de TAN, facilitando o diagnóstico da deficiência auditiva na população infantil. Dentre eles, destaca-se a pesquisa das emissões otoacústicas evocadas (EOE) e o Potencial Evocado Auditivo de Tronco Encefálico (PEATE).

$\mathrm{O} \mathrm{JClH}^{3}$ descreve princípios que visam um programa efetivo de detecção e intervenção o mais cedo possível da perda auditiva e fornece um guia de acompanhamento na implementação e manutenção de um programa de triagem auditiva neonatal bem sucedido, indicando o uso da avaliação fisiológica da audição.

De acordo com o $\mathrm{JCIH}^{3}$, tanto as EOE como o PEATE têm sido usados com sucesso na TANU. No entanto, o documento chama a atenção para o fato de que as EOE detectam somente alterações cocleares e que o PEATE avalia fundamentalmente o nervo auditivo e as vias auditivas do tronco encefálico. Em vista disso, o Grupo de Apoio à Triagem Auditiva Neonatal Universal (GATANU) ${ }^{13}$ recomendou dois tipos diferenciados de protocolos dependendo da presença ou não de indicadores de risco para a deficiência auditiva. No protocolo de baixo risco o GATANU ${ }^{13}$ sugere que seja utilizada numa primeira etapa a pesquisa das EOE, sendo reali- zado PEATE apenas nos bebês que falharam no teste e no reteste com as EOE. Para o protocolo de alto risco, tanto o PEATE como as EOE devem ser utilizados já na primeira etapa.

Outra importante recomendação feita pelo $\mathrm{JCIH}{ }^{2,3}$ diz respeito à necessidade de monitoramento audiológico para todos os bebês que passam na triagem neonatal, mas que têm indicadores de risco associados com perda auditiva de início tardio, progressiva, ou flutuante, bem como desordens da condução neural e/ou disfunção da via auditiva em tronco encefálico, outras desordens auditivas e/ou atraso no desenvolvimento da fala e da linguagem.

$\mathrm{Na}$ tentativa de viabilizar a detecção o mais cedo possível da deficiência auditiva, em 2004, a lei número 14588 - 22/12/2004 foi implementada no estado do Paraná, determinando a obrigatoriedade da TAN em todos os hospitais e maternidades públicos e privados. No entanto, sabe-se que apenas algumas instituições oferecem este serviço, mesmo na capital do Estado, ou seja, em Curitiba.

Desse modo, o objetivo desta pesquisa foi investigar em quais maternidades a Triagem Auditiva Neonatal vem sendo realizada na cidade de Curitiba - PR, bem como qual o protocolo adotado e os resultados obtidos.

\section{MÉTODOS}

A coleta de dados desta pesquisa foi realizada nos meses de maio a agosto no ano de 2006. Inicialmente, foi feito um levantamento do número de maternidades e/ou hospitais com maternidades existentes na cidade de Curitiba mediante a consulta no Cadastro Nacional de Estabelecimentos de Saúde (CNES) ${ }^{15}$, tendo sido constatada a existência de 59 hospitais cadastrados, sendo três desses apenas maternidades e 18 hospitais e maternidades. A partir do levantamento feito, foi estabelecido um contato com o profissional responsável por cada um dos estabelecimentos, sendo questionado quanto à existência de um programa de TAN e para aqueles que o apresentavam foi entregue um questionário a fim de obter informações a respeito do referido serviço. $O$ questionário utilizado constou de 15 perguntas abertas e uma pergunta fechada (Figura 1), elaborado fundamentalmente com base na Declaração de Posição do JCIH (2000) ${ }^{2}$, e foi respondido pelo fonoaudiólogo responsável pelo programa de triagem auditiva.

O presente estudo foi aprovado pelo Comitê de Ética em Pesquisa Institucional sob o parecer número 017/2006. Todos os responsáveis pelos serviços de TAN assinaram um Termo de Consentimento Livre e Esclarecido após terem sido devidamente esclarecidos quanto aos objetivos da pesquisa. 


\section{QUESTIONÁRIO}

Nome da Instituição:

( ) pública ( ) privada

Profissional responsável:

Telefone para contato:

e-mail:

1. Há quanto tempo existe o programa de Triagem Auditiva Neonatal (TAN) nessa maternidade/ hospital?

2. É realizada TAN universal (TANU)?

( ) $\operatorname{sim}($ ) não

3. Se realizada a TANU, qual a porcentagem de bebes triados?

Neonatos de risco:

Neonatos sem risco:

4. Qual a metodologia utilizada para realizar a TAN/TANU (protocolo utilizado) no teste e reteste? Neonatos de Risco:

Neonatos sem risco:

5. Qual é a média de crianças triadas por mês?

Neonatos de risco:

Neonatos sem risco:

6. Se a criança falhar qual o procedimento adotado pelo programa?

Neonatos de risco:

Neonatos sem risco:

7. Com quantas horas de vida do bebê a triagem é realizada?

8. Qual a porcentagem de reteste?

Neonatos de risco:

Neonatos sem risco:

9. Qual a porcentagem de bebês que passaram no reteste?

Neonatos de risco:

Neonatos sem risco:

10. Qual a porcentagem de bebês que concluíram o diagnóstico audiologico?

Neonatos de risco:

Neonatos sem risco:

11. Qual a porcentagem de bebês com diagnóstico de perda auditiva?

Neonatos de risco:

Neonatos sem risco:

12. Qual o tipo e grau de perda auditiva predominante?

Neonatos de risco:

Neonatos sem risco:

13. Qual o tempo médio de vida dos bebês no diagnóstico?

Neonatos de risco:

Neonatos sem risco:

14. Qual a porcentagem de bebês protetizados?

Neonatos de risco:

Neonatos sem risco:

15. Qual o tempo médio de vida dos bebês na protetização?

Neonatos de risco:

Neonatos sem risco:

16. Qual a porcentagem de bebês em intervenção fonoaudiológica?

Neonatos de risco:

Neonatos sem risco:

17. Qual o tempo médio de vida dos bebês no início da intervenção fonoaudiológica?

Neonatos de risco:

Neonatos sem risco: 
A análise das respostas dos questionários foi realizada de forma qualitativa e quantitativa (análise descritiva).

\section{RESULTADOS}

Mediante os resultados obtidos, constatou-se que das 21 instituições com maternidades existentes na cidade de Curitiba, no período no qual esta pesquisa foi realizada, somente cinco $(23,81 \%)$ realizavam Triagem Auditiva Neonatal (TAN), sendo todas da esfera privada. Em quatro desses estabelecimentos, os responsáveis pelos serviços responderam que realizavam a TAN em todos os neonatos de alto risco $(80 \%)$ e apenas em uma maternidade $(20 \%)$ a TANU era realizada, sendo para o restante ainda opcional.

O tempo de vida do neonato no momento da realização da primeira fase da triagem auditiva variou de 36 a 48 horas de vida, e, no reteste (segunda fase da triagem auditiva) variou de 15 a 30 dias de vida. A segunda fase da triagem auditiva era realizada somente com os bebês que falharam na primeira fase.

$\mathrm{Na}$ Tabela 1 podem ser visualizadas as informações referentes à caracterização dos serviços de triagem auditiva prestados na cidade de Curitiba. Nesta etapa de tabulação dos dados não foram incluídos aqueles referentes à maternidade V (MV), uma vez que esta iniciou a implantação do programa dois meses antes à coleta de dados da presente pesquisa.

No questionário constava, ainda, perguntas relativas ao tempo de vida do bebê no diagnóstico da perda auditiva, tempo de vida na adaptação de aparelho de amplificação sonora individual (AASI) e tempo de vida no início da intervenção fonoaudiológica dos casos com diagnóstico de deficiência auditiva. No entanto, nenhuma das respostas relativas a essas questões foi considerada na tabulação dos dados, uma vez que foram constatadas respostas apoiadas em dados da literatura e não baseadas na realidade do programa de triagem auditiva neonatal implantado nas quatro maternidades. Além disso, os casos de perda auditiva apontados pelos profissionais responsáveis pelos serviços não refletiram a estatística real em função dos inúmeros casos que foram perdidos por não comparecerem ao reteste ou a etapa de diagnóstico audiológico.

Cabe ressaltar que nas maternidades I, II e III, o serviço de triagem auditiva neonatal é coordenado pelo mesmo profissional. De acordo com os dados fornecidos por este profissional o índice de neonatos que foram submetidos à TAN e que não concluíram a etapa de identificação de perda auditiva é de aproximadamente $30 \%$. As maternidades IV e V não souberam informar esse dado.

No que se refere ao protocolo utilizado para a realização da TAN na primeira etapa, as cinco maternidades utilizavam as Emissões Otoacústicas Evocadas por Estímulo Transiente (EOET), sendo que em três delas era feito o complemento com a pesquisa do Reflexo Cócleo Palpebral (RCP) nos bebês de alto risco (Tabela 2).

Com relação ao reteste dos bebês que falharam na primeira etapa da TAN, para recém-nascidos a termo, este era realizado com EOET em todos os programas, e para recém-nascidos de alto risco, uma maternidade utilizava a pesquisa das EOET, três realizavam o PEATE e uma realizava a combinação entre PEATE e EOET (Tabela 2).

\section{DISCUSSÃO}

O Paraná foi o primeiro estado brasileiro a legislar sobre a TAN. Segundo a lei N 13.272, de 22 de agosto de 2001, revogada em 27 de outubro de 2003, tornou-se obrigatória a realização gratuita de EOE nos três primeiros meses de vida em crianças nascidas em hospitais públicos e privados, prevendo também diagnóstico e intervenção para os casos necessários ${ }^{16}$.

Os dados obtidos neste estudo, entretanto, demonstraram que a lei não está sendo cumprida,

Tabela 1 - Demonstrativos das características dos serviços relativos ao programa de triagem auditiva neonatal na cidade de Curitiba - PR

\begin{tabular}{lcccc}
\hline & M I & M II & M III & M IV \\
\hline Tempo de existência do serviço em anos & 1 & 1 & 10 & 6 \\
Média mensal de RN triados & 90 & 110 & 170 & 50 \\
Percentual de RN encaminhados para reteste & $5 \%$ & $3 \%$ & $2 \%$ & $\boldsymbol{\Delta}$ \\
Percentual de RN que compareceram ao reteste & $\bullet$ & $\bullet$ & $\bullet$ & $\boldsymbol{\Delta}$ \\
Percentual de crianças com diagnóstico de perda auditiva & $\mathbf{\Delta}$ & $\mathbf{\Delta}$ & $\mathbf{\Delta}$ & $\boldsymbol{\Delta}$ \\
\hline
\end{tabular}

Legenda: $\mathrm{M}$ - maternidade; $\mathrm{RN}$ - recém nascidos; $\boldsymbol{\Delta}$ Não apresenta dados; $\bullet$ dados imprecisos 
Tabela 2 - Tipos de procedimentos adotados na primeira e segunda etapa (reteste) nos Programas de Triagem Auditiva Neonatal na cidade de Curitiba - PR ( $\mathrm{n}$ total = 5 maternidades)

\begin{tabular}{|c|c|c|}
\hline Procedimentos & $\mathbf{N}$ & $\%$ \\
\hline EOET em RN a termo $^{1}$ & 5 & 100 \\
\hline EOET em RN de $\mathrm{AR}^{1}$ & 2 & 40 \\
\hline EOET+ RCP em RN de $A^{1}$ & 3 & 60 \\
\hline EOET no reteste de RN a termo & 5 & 100 \\
\hline EOET no reteste de RN de AR & 1 & 20 \\
\hline PEATE no reteste de RN de AR & 3 & 60 \\
\hline PEATE+EOET no reteste de RN de AR & 1 & 20 \\
\hline
\end{tabular}

Legenda: ${ }^{1}$ dados referentes à primeira etapa; $\mathrm{RN}$ - recém nascido; AR- Alto risco; EOET- Emissões otoacústicas evocadas por estímulo transiente; RPC- Reflexo Cócleo-Palpebral

tendo em vista que, mesmo na capital do estado, ou seja, em Curitiba, somente cinco das 21 instituições com maternidades, isto é, apenas $23,81 \%$ delas, realizam a TAN. Ressalta-se, entretanto, que apesar dessa lei não estar sendo cumprida na maioria das instituições com maternidades existentes na cidade de Curitiba, a mesma pode ter influenciado a implantação dos serviços de TAN existentes, considerando-se que três das cinco instituições com maternidades que realizavam a TAN na época em que esta pesquisa foi realizada ofereciam esse serviço há, no máximo um ano (Tabela 1). Deste modo, os resultados obtidos neste estudo divergem dos mencionados por outros autores ${ }^{16}$ que afirmaram que as leis existentes a respeito da TAN no Brasil parecem não ter influenciado na criação dos serviços de TAN.

No que se refere à Triagem Auditiva Neonatal Universal, apesar da mesma já ter sido recomendada internacionalmente desde $1994{ }^{14}$, apenas uma instituição com maternidade da cidade de Curitiba afirmou que estava realizando esse tipo de procedimento. Além disso, dentro dos pontos de referência dos programas de TANU, o $\mathrm{JCIH}^{3}$ preconizou que, para que a TAN seja eficiente, num período de seis meses após o início do programa, pelo menos 95\% dos recém nascidos devem ser triados. Contudo, os resultados obtidos neste estudo demonstraram que a implantação de programas de Triagem Auditiva Neonatal não vem acompanhando a demanda de nascimentos na cidade de Curitiba-PR.

Em seu último registro realizado em 2005, o DATA SUS ${ }^{17}$ apresentou 33.543 nascidos vivos no município de Curitiba, ou seja, uma média de 2.795 crianças nascidas ao mês. Nesse estudo constatouse que, em média, 420 crianças foram triadas mensalmente, considerando os dados apontados pelas quatro maternidades (Tabela 1), ou seja, somente $15,02 \%$ (5040) do total de nascidos foram submetidos à triagem auditiva. Deste modo, percebe-se que é preciso ampliar e aperfeiçoar os serviços de TAN para abranger toda essa demanda e atingir, conforme preconizado pelo $\mathrm{JCIH}^{3}$, os $95 \%$ do total de nascidos vivos que devem ser submetidos à TAN até o primeiro mês de vida.

Cabe ressaltar que para implementar a TANU neste país existem inúmeras dificuldades ou barreiras a transpor. A associação de fatores como o tamanho territorial do Brasil com suas diferenças socioeconômicas e culturais, escassez de profissional qualificado e recursos financeiros específicos para a realização da TAN, ausência de um sistema de informação para o gerenciamento dos dados, dentre outros, contribuiu para o entendimento de que a TANU deve ter sua implantação gradativa. Tal posicionamento se justifica, principalmente, pautado na prerrogativa de que todos os bebês que forem submetidos à TAN devem ter garantia de acesso ao diagnóstico da alteração auditiva detectada na etapa da triagem auditiva e consequentemente à intervenção terapêutica caso a deficiência auditiva seja confirmada.

Apesar dessas dificuldades, sabe-se que o custo-benefício do diagnóstico da perda auditiva e intervenção até o sexto mês de vida da perda auditiva justificam a implantação de programas de TAN em nível nacional. Por meio destes, a criança tem a possibilidade de ter um desenvolvimento auditivo adequado, bem como, desenvolver a linguagem oral de forma significantemente melhor do que aquelas identificadas tardiamente ${ }^{4-7}$.

No entanto, para atender essa grande demanda de forma efetiva, são necessários recursos financeiros que para um país em desenvolvimento como o Brasil, onde o serviço público não provê assistência imediata e não possui verba suficiente, a execução de um modelo ideal de TAN ainda é um obstáculo e considerado pelos profissionais da área um grande problema de saúde pública. 
A falta de informação e conhecimento da população sobre o assunto ${ }^{18}$ bem como, dos profissionais da área ${ }^{19-22}$ e a pouca valorização da prevenção primária e secundária também podem ser fatores contribuintes para a dificuldade de concretização desse quadro no país ${ }^{23}$.

Em relação ao tempo de vida no qual a TAN é realizada, constatou-se que todos os programas existentes na cidade de Curitiba realizavam a TAN com, no mínimo 36 horas de vida, provavelmente na tentativa de evitar uma porcentagem muito grande de retestes. Essa hipótese foi levantada com base nos estudos que não recomendam a realização da TAN com menos do que 24 horas de vida do bebê em função da maior probabilidade de presença de vérnix no conduto auditivo externo ${ }^{24}$.

Além disso, as porcentagens de reteste encontradas neste estudo (Tabela 1) também corroboram a hipótese levantada acima, tendo em vista que os índices informados pelos profissionais responsáveis pelos programas nas maternidades I, II e III são baixos quando comparados aos $6.1 \%{ }^{10} ; 13 \%$, $11 \%, 8.1 \%{ }^{25}$ reportados em estudos anteriores. Ressalta-se, entretanto, que o profissional responsável pelo programa de triagem auditiva neonatal na MIV, pareceu não ter essa mesma preocupação, pois não soube informar qual o índice de reteste, apesar dos seis anos de existência do mesmo.

Como a falha na TAN pode causar ansiedade nos pais até o momento que o diagnóstico efetivo seja realizado ${ }^{26,27}$, considera-se de extrema importância que os profissionais responsáveis pelos programas de TAN tenham um controle rigoroso dos percentuais de reteste, bem como que esses percentuais sejam os mais baixos possíveis. Além disso, um percentual de reteste baixo pode diminuir o custo-efetividade dos programas de TAN ${ }^{27}$, tendo em vista que um índice de reteste elevado pode inviabilizar a execução das etapas seguintes da TAN, ou seja, diagnóstico e intervenção.

No questionário constava, ainda, perguntas relacionadas ao percentual de recém-nascidos que compareceu ao reteste, percentual de crianças com diagnóstico de perda auditiva e questões direcionadas a etapa de intervenção fonoaudiologica nos casos identificados. No entanto, os profissionais responsáveis pelos serviços apresentaram dados imprecisos ou revelaram não possuir esses dados.

Esses resultados obtidos na presente pesquisa reforçam a necessidade da existência de um sistema de informação para gerenciamento desses dados, e dessa forma, colaborar para a obtenção da prevalência nacional de perda auditiva identificada a partir de programas de TAN, e estabelecer, assim, o perfil epidemiológico do país. A dificuldade em se obter esses dados não se restringe ao Brasil, sendo considerada uma problemática mesmo em paises desenvolvidos ${ }^{3}$.

Além da problemática relacionada à obtenção de dados em nível nacional, existem relatos de dificuldades na implantação da etapa de intervenção fonoaudiologica. De acordo com estudos anteriores, dos indivíduos diagnosticados com perda auditiva, apenas $64 \%$ fazem uso de aparelho de amplificação sonora individual (AASI) e desses, apenas $68 \%$ estão em acompanhamento, porém não se encontra referência ao acompanhamento indicado pela Portaria 587 do SUS, apontando falhas no processo de adaptação e acompanhamento dos usuários ${ }^{28}$.

É importante esclarecer que se não houver adaptação adequada de AASI com devido acompanhamento, associada à terapia fonoaudiológica, o empreendimento financeiro voltado à implementação de programas de Triagem Auditiva Neonatal não é justificável.

Com relação ao procedimento utilizado na realização da TAN as EOET foram predominantes nessa pesquisa (Tabela 2) e é também apontada em muitos trabalhos ${ }^{29-33}$, sendo indicada por ser um exame objetivo, rápido e de fácil aplicação, tendo em vista que a maioria das patologias causadoras de perda auditiva sensório-neural, associadas à surdez infantil, lesionam inicialmente as células ciliadas externas ${ }^{34}$ que são avaliadas por meio deste exame. Porém, alterações retrococleares ou a neuropatia/dessincronia auditiva não são identificadas por meio desse procedimento e são frequentemente encontradas em bebês de alto risco ${ }^{35}$. Portanto, a utilização do PEATE também é necessária, principalmente na avaliação dessa população ${ }^{13}$. Destaca-se que quatro das cinco maternidades que participaram deste estudo realizam 0 PEATE no reteste de bebês de risco. No entanto, se o PEATE somente for realizado nos casos em que os bebês falharam na pesquisa das EOET, não serão detectados os casos de neuropatia/dessincronia auditiva sem alterações de orelha média e/ ou externa associados.

\section{CONCLUSÃO}

A TAN é realizada em apenas $23,81 \%$ das maternidades de Curitiba, não sendo universal na maioria dos serviços existentes, apesar da existência de uma lei que obriga a realização da TAN em todo o estado do Paraná.

$O$ procedimento adotado em todas as maternidades para realização da TAN é o exame de EOET, sendo o PEATE empregado apenas no reteste dos bebês de risco em quatro das cinco maternidades que participaram deste estudo. 
Em relação aos resultados obtidos nos programas de TAN, constatou-se que a maioria das maternidades apresenta um controle do percentual de retestes. No entanto, os dados relativos ao diagnóstico e acompanhamento dos bebês com defici- ência auditiva são inconsistentes, pois não há um sistema de informação que possibilite o gerenciamento dos dados para a obtenção da prevalência de perda auditiva em neonatos saudáveis ou de risco para a deficiência auditiva.

\begin{abstract}
Purpose: to check the practice of newborn hearing screening in Curitiba-PR. Methods: a survey was initially conducted to check the number of existing maternities and hospitals with maternities included in the National Register of Health's Establishments. We found that 59 hospitals were registered; three are maternities and 18 are hospitals that include a maternity. We asked each of the professionals in charge for the institution if some kind of newborn hearing screening was conducted and, for those who had the service, a questionnaire was given in order to collect information on the program. Results: we found that $23.8 \%$ of the institutions perform newborn hearing screening. In $20 \%$ of them, this service is universal for full term babies, while in $80 \%$ of them it is available for premature/high risk babies. All of the services use Transient Evoked Otoacoustic Emissions (TEOAE). Conclusion: we concluded that the Brazilian law number $14588-22 / 12 / 2004$ is not being followed. Despite of the need and the importance for early detection of hearing loss, hearing screening is not accomplished in all maternities. In places where such screening takes place, it is not universal.
\end{abstract}

KEYWORDS: Hearing; Hearing Loss; Neonatal Screening

\section{REFERÊNCIAS}

1. Guarinelo AC, Massi G, Berberian AP. Surdez e linguagem escrita: um estudo de caso. Rev Bras Educ Espec. 2007; 13(2):205-18.

2. Joint Committee on Infant Hearing. Year 2000 Position statement: principles and guidelines for early hearing detection and intervention programs. Pediatrics. 2000; 106(4):798-817.

3. Joint Committee on Infant Hearing. Year 2007 Position statement: principles and guidelines for early hearing detection and intervention programs. Pediatrics. 2007; 120(4):898-921.

4. Yoshinaga-Itano C, Sedey AL, Coulter DK, Mehl AL. Language of early- and later-identified children with hearing loss. Pediatrics. 1998; 102(5):1161-71.

5. Yoshinaga-Itano C, Coulter D, Thomson V. The Colorado newborn hearing screening project: effects on speech and language development for children with hearing loss. J Perinatol. 2000; 20(8S):S132-7.

6. Yoshinaga-Itano C, Coulter D, Thomson V. Developmental outcomes of children with hearing loss born in Colorado hospitals with and without universal newborn hearing screening programs. Semin Neonatol. 2001; 6(6):521-9.

7. Yoshinaga-Itano C. Early intervention after universal neonatal hearing screening: impact on outcomes. Ment Retard Dev Disabil Res Rev. 2003; 9(4):252-66.

8. Nóbrega M. Triagem audiológica universal. In: Caldas N, Caldas SN, Sih T. Otologia e audiologia em pediatria. Rio de Janeiro: Revinter; 1999. p. 208-10.

9. Ramos BD. Importância da audição no desenvolvimento da linguagem. In: Caldas N, Caldas $\mathrm{SN}$, Sih T. Otologia e audiologia em pediatria. Rio de Janeiro: Revinter; 1999. p.168-71.

10. Durante AS, Carvallo RMM, Costa MTZ, Cianciarullo MA, Voegels RL, Takahashi GM, et al. Programa de triagem auditiva neonatal: modelo de implementação. Arq Int Otorrinolaringol. 2004; 8(1):56-71.

11. Pádua FGM, Marone S, Bento RF, Carvallo RMM, Durante AS, Soares JC, et al. Triagem auditiva neonatal: um desafio para sua implantação. Arq Int Otorrinolaringol. 2005; 9(3):190-4.

12. Borges CAB, Moreira LMO, Pena GM, Fernandes FR, Borges BCB, Otani BH. Triagem auditiva neonatal universal. Arq Int Otorrinolaringol. 2006; 10(1):28-34.

13. Grupo de Apoio à Triagem Auditiva Neonatal Universal (GATANU). Triagem. [homepage na internet]. São Paulo: GATANU; 2005. [acesso em 20 de jun 2007] Disponível em: URL: http://www. gatanu.org/tan/protocolos.php. 
14. Joint Commitee on Infant Hearing. 1994 Position statement. Audiology Today. 1994; 6(6):6-9.

15. Cadastro Nacional de Estabelecimentos de Saúde (CNES). [homepage na internet] Rio de Janeiro: CNES; 2000 [acesso em 03 de jul 2007] Disponível em: URL: http://cnes.datasus.gov.br.

16. Tochetto T, Vieira PE. Legislação brasileira sobre triagem auditiva neonatal. São Paulo: PróFono; 2006.

17. Brasil. Ministério da Saúde - Data SUS. [homepage na internet] Brasília: Ministério da Saúde; 2008. [acesso em 03 de jul 2007] Disponível em: URL: http://tabnet.datasus.gov.br/cgi/tabcgi. exe?sinasc/cnv/nvpr.def.

18. Ribeiro FG, Mitre E. Avaliação do conhecimento sobre triagem auditiva neonatal de pacientes no pós-parto imediato. Rev. CEFAC. 2004; 6(3):294-9. 19. Barros ACT, Galindo MAC, Jacob RTS. Conhecimento e conduta de pediatras frente à deficiência auditiva. Pediatria. 2002; 24(1/2):25-31 20. Tschiedel RS, Bandini HHM, Bevilacqua MC. Diagnóstico da deficiência auditiva na infância: uma avaliação do nível de conhecimento dos pediatras de uma cidade da região do Centro-Oeste paulista. Pediatr Mod. 2000; 36(9):600-18.

21. Hilú MRPB, Zeigelboim, BS. O conhecimento, a valorização da triagem auditiva neonatal e a intervenção precoce da perda auditiva. Rev. CEFAC. 2007; 9(4):563-70. dx.doi.org/ S1516-18462007000400017

22. Zocoli AMF, Riechel FC, Zeigelboim BS, Marques JM. Audição: abordagem do pediatra acerca dessa temática. Rev Bras Otorrinolaringol. 2006; 72(5):617-23.

23. Gatto $\mathrm{Cl}$, Tochetto TM. Deficiência auditiva infantil: implicações e soluções. Rev CEFAC. 2007; 9(1):110-5. dx.doi.org/S1516-18462007000100014 24. Korres S, Nikolopoulos T, Ferekidis E, Gotzamanoglou Z, Georgiou A, Balatsouras DG. Otoacoustic emissions in universal hearing screening: which day after birth should we examine the newborns? J Oto-Rhino-Laryngol. 2003; 65(4): 199-201.

25. Barreira-Nielsen C, Futuro Neto HA, Gattaz G. Processo de implantação de Programa de Saúde
Auditiva em duas maternidades públicas. Rev. Soc. Bras. Fonoaudiol. 2007; 12(2):99-105.

26. Poulakis Z, Barker M, Wake M. Six month impact of false positives in an Australian infant hearing screening programme. Arch Dis Childhood. 2003; 88(1):20-4.

27. Marques TR, Mendes PC, Bochnia CFP, Jacob LCB, Roggia SM, Marques JM. Triagem auditiva neonatal: relação entre banho e índice de reteste. Rev Bras Otorrinolaringol. 2008; 74(3):375-81.

28. Tschoeke SN. Estudo da deficiência auditiva: características de diagnóstico, etiologia e (re)habilitação [dissertação]. Curitiba (PR): Universidade Tuiuti do Paraná; 2006.

29. Durante AS, Carvallo RMM, Costa FS, Soares JC. Características das emissões otoacústicas por transientes em programa de triagem auditiva neonatal. Pró-Fono. 2005; 17(2):133-40.

30. Bassetto MCA, Chiari BM, Azevedo MF. Emissões otoacústicas evocadas transientes (EOAET): amplitude da resposta em recémnascidos a termo e pré-termo. Rev Bras Otorrinolaringol. 2003; 69(1):84-92.

31. Martines F, Porrello M, Ferrara M, Martines M, Martines E. Newborn hearing screening project using transient evoked otoacoustic emissions: Western Sicily experience. Int J Pediatr Otorhinolaryngol. 2007; 71(1):107-12.

32. White KR, Vohr BR, Meyer S, Widen JE, Johnson JL, Gravel JS, et al. A multisite study to examine the efficacy of the otoacoustic emission/automated auditory brainstem response newborn hearing screening protocol: research design and results of the study. Am J Audiol. 2005; 14(2):S186-99.

33. Johnson JL, White KR, Widen JE, Gravel JS, Vohr BR, James M, et al. A multisite study to examine the efficacy of the otoacoustic emission/automated auditory brainstem response newborn hearing screening protocol: introduction and overview of the study. Am J Audiol. 2005; 14(2):S178-85.

34. Oliveira P, Castro FE, Ribeiro A. Surdez infantil. Rev Bras Otorrinolaringol. 2002; 68(3):417-23.

35. Rea PA, Gibson WP. Evidence for surviving outer hair cell function in congenitally deaf ears. Laryngoscope. 2003; 113(11):2030-4.

RECEBIDO EM: 26/04/08

ACEITO EM: 27/04/2009

Endereço para correspondência:

Candice Cristina Stumpf

Rua Salgado Filho, 394

Joaçaba - SC

CEP: 89600-000

E-mail: candicestumpf@ hotmail.com 